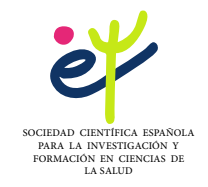

European Journal of Health Research

2021, Vol. 7, No 2 (Págs. 1-19)

Eur. J. Health. Research. e-ISSN 2445-0308

https://revistas.uautonoma.cl/index.php/ejhr

doi: $10.32457 /$ ejhr.v7i2.1592

\title{
Nomograma de predicción para la estratificación del riesgo en pacientes con COVID-19
}

\section{Prediction nomogram for risk stratification in patients with COVID-19}

\author{
Carlos E. Herrera Cartaya ${ }^{* 1,2}$ (D), Agustín Lage Dávila ${ }^{3}$ (D, \\ Julio Betancourt Cervantes ${ }^{2}$ D, Eligio E. Barreto Fiu ${ }^{4}$ iD, Lizet Sánchez Valdés ${ }^{3}$ \\ y Tania Crombet $\operatorname{Ramos}^{3}$ (D) \\ ${ }^{1}$ Departamento de Medicina Intensiva. Hospital Universitario Arnaldo Milián Castro. Villa Clara. \\ Cuba; ${ }^{2}$ Departamento de Medicina Intensiva. Hospital Universitario Manuel Fajardo. Villa Clara. \\ Cuba; ${ }^{3}$ Centro de Inmunología Molecular. La Habana. Cuba; ${ }^{4}$ Departamento de Bioestadística. Uni- \\ versidad Médica de Villa Clara. Cuba.
}

\begin{abstract}
Resumen
La COVID-19 puede progresar a formas severas de la enfermedad con una elevada mortalidad, por lo que ha sido necesario identificar factores predictivos que permitan estratificar el riesgo en los enfermos. Se realizó un estudio retrospectivo analítico en una cohorte de 150 pacientes del hospital Manuel Fajardo de Villa Clara, Cuba, en el periodo de marzo a junio de 2020. Con la información obtenida se construyó un índice pronóstico de severidad mediante un modelo de regresión logística binaria multivariada, en el que se expresó la probabilidad de que el paciente evolucionara hacia la severidad en función del conjunto de variables que fueron identificadas como predictoras del evento de salud de interés. Para el resumen de los datos y la aplicación de las pruebas de hipótesis se utilizó el software $\mathrm{R}$ versión 4.0.2. Con los resultados finales se elaboró un índice pronostico a través de una ecuación matemática en la cual se sustenta el modelo. Para facilitar su interpretación se construyó el nomograma de predicción, que constituyó la salida principal de este estudio. Las variables con mayor poder predictivo, que definitivamente quedaron en el modelo y con las cuales se construyó el nomograma fueron: edad $(\mathrm{p}=0.049)$, hipertensión arterial $(\mathrm{p}=0.013)$, índice neutrófilos/linfocitos $(\mathrm{p}=0.004)$, deshidrogenasa láctica $(\mathrm{p}=0.039)$ y saturación arterial de oxígeno $(\mathrm{p}=0.044)$. El resultado del estadígrafo del test de Hosmer-
\end{abstract}

* Autor de correspondencia: Carlos. E. Herrera Cartaya. Dirección: Avenida Hospital y Doble Vía. Santa Clara. Villa Clara. Cuba. Correo electrónico: carloshc@infomed.sld.cu 
Lemeshow fue $\mathrm{p}=0.976$ y la capacidad discriminatoria dada por el área bajo la curva ROC (receiver operating characteristic curve) fue igual a 0.988 (AUC: $0.9882,95 \% \mathrm{CI}$ : 0.9756-1). El punto de corte óptimo fue 0. 099.Se concluye que nuestro nomograma constituye un instrumento de gran utilidad para identificar precozmente los pacientes con riesgo de progresar a formas severas de COVID-19. De esta manera, facilita una mejor estratificación y adecuación de tratamientos oportunos, capaces de frenar la progresión de la enfermedad.

Palabras clave: COVID-19, COVID-19 severo, pronóstico, riesgo, nomograma.

\section{Summary}

COVID-19 can progress to severe forms of the disease with high mortality, so it has been necessary to identify predictive factors that allow stratifying the risk in patients. A retrospective analytical study was carried out in a cohort of 150 patients from Manuel Fajardo Hospital in Villa Clara, Cuba, from March to June 2020. With the information obtained, a severity prognostic index was constructed by means of a multivariate binary logistic regression model, in which the probability of the patient evolving towards severity was expressed as a function of the set of variables that were identified as predictors of the health event of interest. $\mathrm{R}$ software version 4.0.2 (2206-2020) was used to summarize the data and apply the hypothesis tests. With the final results, a prognostic index was elaborated through a mathematical equation on which the model is based. A prediction nomogram was constructed to facilitate its interpretation, which constituted the main output of this study. The variables with the highest predictive power, which definitely remained in the model and with the nomogram was constructed were: age $(\mathrm{p}=0.049)$, arterial hypertension $(\mathrm{p}=0.013)$, neutrophil/lymphocyte ratio $(\mathrm{p}=0.004)$, lactate dehydrogenase $(\mathrm{p}=0.039)$ and arterial oxygen saturation $(\mathrm{p}=0.044)$. The Hosmer-Lemeshow test statistic result was $\mathrm{p}=0.976$ and the discriminatory capacity given by the area under the ROC curve (receiver operating characteristic curve) was equal to 0.988 (AUC: 0.9882, 95\% CI: 0.9756-1). The optimal cut-off point was 0.099 . We conclude that our nomogram is a very useful tool for the early identification of patients at risk of progressing to severe forms of COVID-19. In this way, it facilitates better stratification and the adequacy of timely treatment, capable of slowing disease progression.

Key words: COVID-19, severe COVID-19, prognosis, risk, nomogram.

\section{INTRODUCCIÓN}

La enfermedad por COVID-19 causada por el nuevo coronavirus, síndrome respiratorio agudo severo-coronavirus-2 (SARSCoV-2), ha sido un reto para la salud pública mundial. 
El 12 de diciembre de 2019 fue detectado un brote de neumonía de etiología no precisada en la ciudad de Wuhan, China. Desde entonces los sistemas sanitarios de los países fueron puestos en alerta. El 11 de marzo de 2020 la Organización Mundial de la Salud anunciaba la COVID-19 como una pandemia global con un alcance impredecible y posibles consecuencias devastadoras para la humanidad (Ji et al.2020; Lu et al., 2020).

Para disminuir el daño asociado a la COVID-19, los sistemas de salud pública y control de infecciones han requerido tomar medidas urgentes para limitar la extensión global del virus y sus consecuencias (Song, 2020).

La COVID-19 es una enfermedad que puede tener un curso leve o asintomático, pero en su evolución puede progresar a formas graves, e incluso enfermedad crítica, con una elevada mortalidad. Esta fase se caracteriza por la aparición de síndrome de distress respiratorio agudo y fallo múltiple de órganos. Está clara la necesidad de optimizar la atención médica de los pacientes en aras de la asignación racional de recursos, unido a una mayor efectividad de los tratamientos. Por lo tanto, ha sido necesario con urgencia identificar factores pronósticos, tanto clínicos como paraclínicos, que permitan estratificar el riesgo en los enfermos y controlar activamente la gravedad de la enfermedad (Vélez et al., 2020).

Datos epidemiológicos sugieren que el rango de complicaciones y mortalidad en pacientes con COVID-19 severa es mayor que en pacientes sin manifestaciones de severidad (Chen et al., 2020; Zhou et al., 2020; Yang et al, 2020).

En aras de mitigar la carga de los sistemas de salud. y a la vez brindar el mejor cuidado posible a los pacientes, se necesita una información eficiente de su pronóstico. En el ámbito médico ha existido un interés global en el desarrollo de modelos predictivos para la COVID-19. Estos incluyen investigaciones diseñadas con el objetivo de predecir el diagnóstico, así como el curso de la enfermedad (Wynants et al., 2020).

La rápida, efectiva clasificación, y estratificación de los pacientes atendiendo a sus características, garantiza a los de mayor riesgo, una adecuada monitorización y manejo. Esta información permite realizar recomendaciones personalizadas sobre tratamientos en determinados grupos. Los modelos pronósticos pueden tener un valor adicional en dicha estratificación (Beigel et al., 2020; Horby et al., 2021).

Los modelos predictivos de severidad combinan diversas variables o características que permiten estimar en la población enferma el riesgo de un peor pronóstico durante la infección. Estos pueden asistir al equipo médico para clasificar los pacientes según sus riesgos, en aras de un adecuado resultado. Sistemas de escalas de puntuación, así como modelos de aprendizaje automático avanzado han sido propuestos en respuesta a la necesidad de investigación y divulgación de resultados relevantes sobre esta enfermedad; todo con el objetivo de contribuir a la necesidad de mejorar la atención e incrementar el número de vidas salvadas (Wynants et al., 2020; Lipsitch et al., 2020). 
En nuestra experiencia la identificación precoz de pacientes con riesgo de evolucionar a formas severas de la COVID-19 nos ha facilitado una mejor estratificación, y dirigir determinados tratamientos hacia aquellos grupos con mayor probabilidad de agravarse. En tal sentido desarrollamos un modelo predictivo que incluye la construcción de un nomograma de riesgo, el cual clasifica de manera eficiente la probabilidad de severidad.

\section{MÉTODO}

La construcción del nomograma parte de un estudio retrospectivo analítico en una cohorte de 150 pacientes del hospital Manuel Fajardo de Villa Clara, Cuba, que ingresaron con el diagnóstico de COVID-19 según los resultados de la prueba de reacción a la polimerasa $(P C R)$ en tiempo real. El período del estudio estuvo comprendido desde marzo a junio de 2020 .

Los pacientes fueron asignados a dos grupos. Teniendo en cuenta la evolución clínica y el ingreso en la Unidad de Cuidados Intensivos como criterio de severidad, fueron agrupados de la siguiente manera:

Grupo no severo $(\mathrm{n}=124)$ : Pacientes con evolución satisfactoria que no ingresaron en la unidad de cuidados intensivos (UCI).

Grupo severo ( $n=26)$ : Pacientes con necesidad de ingreso en la UCI.

\section{Recolección de datos y análisis estadístico}

Para la recolección de los datos se tuvieron en cuenta variables demográficas, clínicas, de laboratorio, gasométricas y radiológicas. Estas fueron obtenidas en estado basal de los pacientes luego del ingreso en el hospital.

Variables demográficas y clínicas: Edad, sexo, comorbilidad, manifestaciones clínicas y puntuación de la escala pronóstica Quick SOFA.

Variables de laboratorio: Hemoglobina $(\mathrm{g} / \mathrm{dL})$, leucocitos totales $\left(\mathrm{x} 10^{9} / \mathrm{L}\right)$, neutrófilos (\%), linfocitos $(\%)$, plaquetas $(\times 10 \% / \mathrm{L})$, índice neutrófilos/linfocitos (INL) obtenido de la división del total de neutrófilos entre el total de linfocitos, glicemia $(\mathrm{mmol} / \mathrm{L})$, creatinina $(\mu \mathrm{mol} / \mathrm{l})$, lactato $(\mathrm{mmol} / \mathrm{L})$, lactato deshidrogenasa $(\mathrm{LDH}, \mathrm{mmol} / \mathrm{L})$, alaninaaminotranferasa (ALT, UI), Proteína C reactiva (+/-), dimero D $(\mu \mathrm{g} / \mathrm{L})$; electrolitos: sodio, potasio, cloro y calcio $(\mathrm{mmol} / \mathrm{L})$.

Variables gasométricas: $\mathrm{pH}$, presión arterial de oxígeno $\left(\mathrm{PaO}_{2}, \mathrm{mmHg}\right)$, presión arterial de dióxido de carbono $\left(\mathrm{PaCO}_{2} \mathrm{mmHg}\right)$, saturación arterial de oxígeno $\left(\mathrm{SaO}_{2}, \%\right)$, bicarbonato $\left(\mathrm{HCO}_{2}, \mathrm{mmol} / \mathrm{l}\right)$, exceso de bases $(\mathrm{BB},+/-, \mathrm{mmol} / \mathrm{L})$.

Radiológicas: Presencia de Infiltrados o consolidación pulmonar. 
Los datos fueron obtenidos a partir de la información de las historias clínicas y los resultados de los exámenes radiológicos y de laboratorio.

A partir de la información obtenida se construyó un índice pronóstico de severidad mediante la utilización de un modelo de regresión logística binaria multivariada, en el que se expresó la probabilidad de que el paciente evolucionara hacia el estado de severidad en función del conjunto de variables que fueron identificadas como predictores del evento de salud de interés (severidad). Para el resumen de los datos y la aplicación de las pruebas de hipótesis se utilizó el software libre R versión 4.0.2 (22-06-2020).

El análisis estadístico se realizó en función de diferentes etapas a través de las cuales se obtuvo el modelo:

Etapa 1: Análisis bivariado para la comparación de los grupos (severo y no severo) según las variables de estudio.

La identificación de los predictores comenzó con la comparación de los grupos, (severo vs no severo) a través de pruebas estadísticas que fueron seleccionadas según la escala de medición de las variables, así como su distribución. Aquellas en las que los grupos mostraron diferencias significativas, fueron incluidas en la segunda etapa.

Etapa 2: Medición de la asociación entre cada una de las variables con diferencias significativas entre los grupos y la severidad de la enfermedad.

Se determinó como medida de asociación, la razón de oportunidades u $\operatorname{Odds}$ Ratio $(O R)$, mediante la aplicación de la regresión logística binaria simple. Se consideró a una variable con $O R$ significativa, cuando el valor de uno no pertenece al intervalo de confianza (95\%) para la misma. Aquellas con un valor de $\mathrm{p}<0.05$ pasaron a la tercera etapa.

Etapa 3: Reducción de la dimensionalidad de los datos correspondientes a variables con $O R$ significativa.

Para reducir el número de variables que continuaron en el estudio fueron utilizados dos procedimientos adicionales:

1. La regresión LASSO (Least Absolute Shrinkage and Selection Operator)

2. El método de selección automática hacia atrás (Backward)

Esta reducción fue realizada con la finalidad de evitar "sobreajuste" (overfitting) en las estimaciones de los parámetros de un modelo de regresión, así como la multicolinealidad de las variables explicativas que también pueden afectar la precisión de las mismas.

Las variables se incluyeron en la regresión $L A S S O$ teniendo en cuenta el hiperparámetro de penalización lambda $(\lambda)$, que se determinó en primera instancia, a partir de la muestra y del procedimiento de validación cruzada.

Las que resultaron con coeficientes diferentes de cero, se les aplicó posteriormente el método de selección automática hacia atrás (Backward). Se introdujeron todas las variables 
en un primer paso, y luego se comenzó a excluir una tras otra según el contraste individual basado en el estadístico Z, de manera tal que fueron eliminadas las menos influyentes.

Ambos procedimientos fueron aplicados en el contexto de una regresión logística binaria multivariada.

Etapa 4: Construcción del nomograma de predicción.

Para evaluar el rendimiento de este índice se realizó la validación aparente, la que se obtuvo a partir del test de Hosmer-Lemeshow y la determinación de su poder de discriminación según el área bajo la curva $R O C$ (receiver operating characteristic curve) y el punto de corte óptimo.

Con los resultados finales se elaboró un índice pronóstico a través de una ecuación matemática en la cual se sustenta el modelo. Para facilitar su interpretación se construyó el nomograma de predicción, que constituyó la salida principal de este estudio.

\section{RESULTADOS}

En la tabla I se muestra para cada variable independiente o posible variable predictiva, el valor estimado de la $O R$, con su significación (p) asociado al contraste cuya hipótesis nula se plantea como $(\mathrm{Ho}: O R=1)$, interpretado como falta de asociación. También se muestra el intervalo de confianza para la $O R$.

\section{Tabla I.}

Resultados de la regresión logistica binaria simple.

\begin{tabular}{lccc}
\hline Variable & OR & $\mathrm{P}$ & IC $(95 \%)$ \\
\hline Edad & 1.09 & $<0.0001$ & $1.06-1.13$ \\
HTA & 7.18 & $<0.0001$ & $2.77-18.62$ \\
Diabetes mellitus & 7.12 & $<0.0001$ & $2.57-19.78$ \\
Demencia & 7.14 & 0.0026 & $1.99-25.62$ \\
EPOC & 7.33 & 0.0126 & $1.53-35.05$ \\
Cáncer & 2.44 & 0.4734 & $0.21-27.96$ \\
Polipnea $(f r \geq 22 \mathrm{rpm})$ & 24.14 & $<0.0001$ & $8.40-69.32$ \\
Diarreas & 7.12 & 0.0002 & $2.57-19.78$ \\
Quick SOFA $\geq 1$ & 25.65 & $<0.0001$ & $8.94-73.61$ \\
Leucocitos x 10 $\% / \mathrm{L}$ & 1.39 & 0.0002 & $1.17-1.65$ \\
Neutrófilos \% & 1.24 & $<0.0001$ & $1.14-1.34$ \\
Linfocitos \% & 0.78 & $<0.0001$ & $0.70-0.86$ \\
INL & 1.83 & $<0.0001$ & $1.48-2.27$ \\
Hb g/dL & 0.56 & $<0.0001$ & $0.43-0.73$ \\
\hline
\end{tabular}




\begin{tabular}{lccc}
\hline Variable & $O R$ & $\mathrm{P}$ & $\mathrm{IC}(95 \%)$ \\
\hline Glicemia mmol/L & 1.01 & 0.7269 & $0.96-1.07$ \\
$\mathrm{LDH} \mathrm{U} / \mathrm{L}$ & 1.01 & $<0.0001$ & $1.01-1.01$ \\
Dímero D ug/L & 3.67 & 0.0021 & $1.60-8.42$ \\
Lactato mmol/L & 12.87 & 0.0012 & $2.73-60.66$ \\
$\mathrm{PrCR}$ positiva & 3.69 & 0.0049 & $1.49-9.14$ \\
Calcio mmol/L & 0.003 & 0.0132 & $0.00-0.29$ \\
$\mathrm{PaCO}_{2} \mathrm{mmHg}$ & 0.85 & 0.0075 & $0.76-0.96$ \\
$\mathrm{PaO}_{2} \mathrm{mmHg}$ & 0.91 & 0.0018 & $0.86-0.97$ \\
$\mathrm{SaO}_{2} \%$ & 0.35 & $<0.0001$ & $0.23-0.52$ \\
$\mathrm{Consolidación} \mathrm{pulmonar}_{\text {Infiltrado pulmonar }}^{32.29}$ & $<0.0001$ & $6.43-162.21$ \\
\hline
\end{tabular}

Leyenda: Significación ( $p$ ). Intervalo de confianza (IC) 95\% de confiabilidad. HTA: Hipertensión arterial. EPOC: Enfermedad pulmonar obstructiva crónica. fr: frecuencia respiratoria. Rpm: respiraciones por minuto. INL: Indice neutrófilos/linfocitos. LDH: Lactato deshidrogenasa. PrCR: Proteina C reactiva. PaCO ${ }_{2}$ : Presión arterial de dióxido de carbono. $\mathrm{PaO}_{2}$. Presión arterial de Oxigeno. $\mathrm{SaO}_{2}$ : Saturación arterial de oxígeno.

En la figura 1 se representa la determinación del valor óptimo del coeficiente de penalización en la regresión LASSO. El grafico A muestra los valores de los coeficientes según el logaritmo del hiperparámetro $\lambda$. A medida que aumenta el logaritmo de $\lambda$, la penalización es mayor, y se contrae el valor de los coeficientes. Algunos coeficientes se hacen cero a partir de ciertos valores de $\lambda$, lo que hace que se reduzca el número de variables. El gráfico $\mathrm{B}$ muestra la penalización óptima según la deviance y el valor del logaritmo del hiperparámetro $\lambda$ A medida que $\lambda$ es mayor, aumenta la deviance (desviación o desajuste) y disminuye el número de variables. Los puntos en rojos representan la deviance para cada modelo construido a partir del valor que se le asigna a $\lambda$, y las líneas en las que se inscriben, constituyen los intervalos de confianza. La línea de vertical de puntos significa el valor óptimo del $\log (\lambda)(-5.445)$ y por ende de $\lambda(0.0043)$, en correspondencia con la deviance mínima. 


\section{Figura 1.}

Resultados de la regresión LASSO. A. Coeficientes de las variables según el logaritmo del hiperparámetro $\lambda$.B. Penalización óptima según deviance y el valor del logaritmo del hiperparámetro $\lambda$.

A

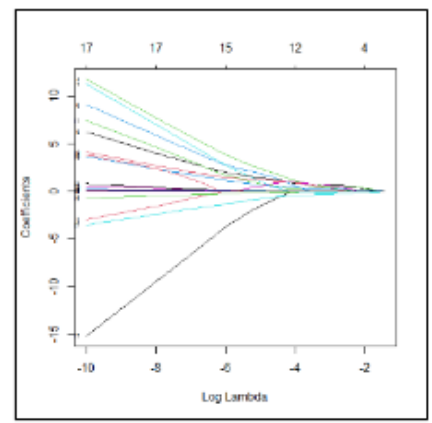

B

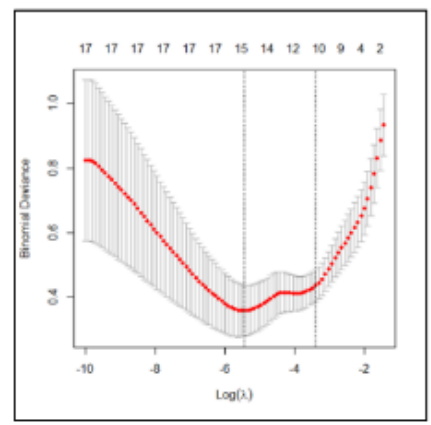

En la tabla II se muestran los valores estimados de los coeficientes en la ecuación del modelo logístico binario multivariado a través del método de regularización o contracción LASSO.

Resultaron con estimación de coeficientes igual a cero las variables: sexo, diabetes mellitus, demencia, EPOC, fiebre, leucocitos, lactato, proteína $\mathrm{C}$ reactiva, calcio, $\mathrm{PaCO}_{2}$, $\mathrm{PaO}_{2}$, e infiltrado pulmonar. Por lo tanto, estas variables salen del modelo predictivo. Se excluyeron del modelo el dímero $\mathrm{D}$ por tener un número de datos faltantes superior al $7 \%$ de la muestra, y el Quick SOFA por ser una escala(Singer et al., 2016), que mide variables que coinciden con otras que se analizan en el modelo.

Quedaron en el modelo las variables: edad, HTA, polipnea, diarreas, INL, hemoglobina, $\mathrm{LDH}, \mathrm{SaO}_{2}$, y consolidación pulmonar. Estas continuaron su análisis en el siguiente paso.

\section{Tabla II.}

Estimaciones de los coeficientes de la regresión logistica LASSO.

\begin{tabular}{lc}
\hline Variables & Coeficiente \\
\hline Edad & 0.0094 \\
Sexo & $\cdot$ \\
HTA & 0.6683 \\
Diabetes mellitus & $\cdot$ \\
Demencia & $\cdot$ \\
EPOC & $\cdot$ \\
\hline
\end{tabular}




\begin{tabular}{|c|c|}
\hline Variables & Coeficiente \\
\hline Fiebre $\left(\right.$ temp $\left.\geq 37.3^{\circ} \mathrm{C}\right)$ & . \\
\hline Polipnea (fr $\geq 22 \mathrm{rpm})$ & 0.7211 \\
\hline Diarreas & 0.5611 \\
\hline Leucocitos $\times 10^{9} / \mathrm{L}$ & . \\
\hline INL & 0.2821 \\
\hline $\mathrm{Hb} g / d L$ & -0.1193 \\
\hline $\mathrm{LDH} \mathrm{U} / \mathrm{L}$ & 0.0015 \\
\hline Lactato $\mathrm{mmol} / \mathrm{L}$ & . \\
\hline PrCR positiva & . \\
\hline Calcio mmol/L & . \\
\hline $\mathrm{PaCO}_{2} \mathrm{~mm} \mathrm{Hg}$ & . \\
\hline $\mathrm{PaO}_{2} \mathrm{~mm} \mathrm{Hg}$ & . \\
\hline $\mathrm{SaO}_{2} \%$ & -0.3543 \\
\hline Consolidación pulmonar & 0.7260 \\
\hline Infiltrado pulmonar & . \\
\hline Intercepto & 30.6427 \\
\hline
\end{tabular}

Leyenda: HTA: Hipertensión arterial. EPOC: Enfermedad pulmonar obstructiva crónica. fr: frecuencia respiratoria. rpm: respiraciones por minuto. INL: Indice neutrófilos/linfocitos. LDH: Lactato deshidrogenasa. PrCR: Proteina C reactiva. $\mathrm{PaCO}_{2}$ : Presión arterial de dióxido de carbono. $\mathrm{PaO}_{2}$; Presión arterial de Oxígeno. $\mathrm{SaO}_{2}$ : Saturación arterial de oxígeno.

La tabla III representa los resultados del método de selección automática de variables Backward. Las variables que definitivamente quedan en el modelo son: edad ( $\mathrm{p}=0.049)$, HTA ( $\mathrm{p}=0.013)$, INL ( $\mathrm{p}=0.004), \mathrm{LDH}(\mathrm{p}=0.039)$ y SaO${ }_{2}(\mathrm{p}=0.044)$.

Tabla III.

Resultados del método de selección automática de variables Backward.

\begin{tabular}{lcccc}
\hline Coeficientes & Estimación & Error estándar & $\mathrm{Z}$ & $\mathrm{p}$ \\
\hline Intercepto & 79.59 & 46.58 & 1.71 & 0.087 \\
Edad & 0.08 & 0.04 & 1.96 & 0.049 \\
$\mathrm{HTA}$ & 4.49 & 1.81 & 2.49 & 0.013 \\
Polipnea (fr $\geq 22 \mathrm{rpm})$ & 1.73 & 1.07 & 1.62 & 0.105 \\
$\mathrm{INL}$ & 0.85 & 0.29 & 2.91 & 0.004 \\
$\mathrm{LDH} \mathrm{U} / \mathrm{L}$ & 0.01 & 0.01 & 2.07 & 0.039 \\
$\mathrm{SaO}_{2} \%$ & -1.03 & 0.51 & -2.01 & 0.044 \\
\hline
\end{tabular}

Leyenda: HTA: Hipertensión arterial. fr: frecuencia respiratoria. rpm: respiraciones por minuto.INL: Indice neutrófilos/ linfocitos. LDH: Deshidrogenasa Láctica. $\mathrm{SaO}_{2}$ : Saturación arterial de oxígeno. 
A partir de los resultados que se presentan se elaboró un índice pronóstico sustentado en la siguiente expresión aritmética:

$$
\text { Probabilidad }(\text { severidad })=\frac{1}{1+e^{-(79.59+0.08 E d a d+4.49 H T A+0.85 I N L+0.01 L D H-1.03 S a O 2)}}
$$

El resultado del estadígrafo del test de Hosmer-Lemeshow fue $\mathrm{p}=0.976$ y la capacidad discriminatoria dada por el área bajo la curva ROC (receiver operating characteristic curve) fue igual a 0.988(AUC: $0.9882,95 \%$ CI: 0.9756-1). El punto de corte óptimo fue 0.099 .

\section{Figura 2.}

Curva ROC para el modelo predictivo de severidad de la COVID-19

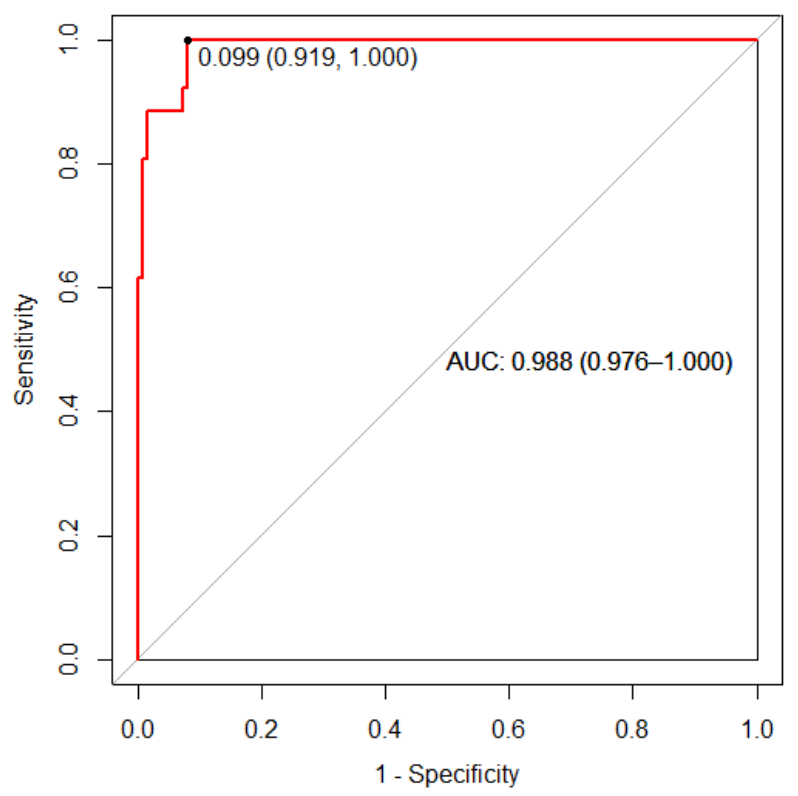

Finalmente, en la figura 3 se muestra el nomograma de predicción de severidad para pacientes con COVID-19, compuesto por las 5 variables que resultaron con mayor poder predictivo. 


\section{Figura 3.}

Nomograma de predicción de severidad de la COVID-19.

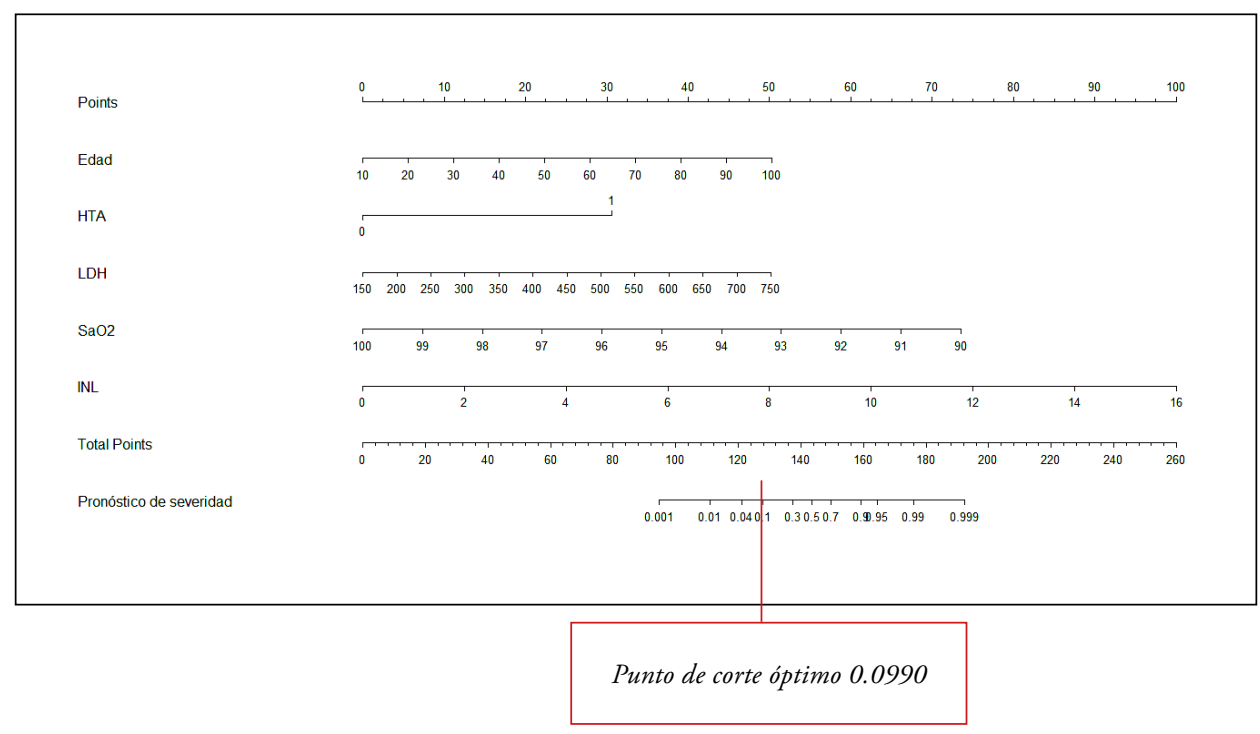

\section{DISCUSIÓN}

Las variables que resultaron con mayor poder predictivo fueron: edad, HTA, LDH, INL y $\mathrm{SaO}_{2}$; las cuales fueron representadas en el nomograma de predicción de riesgo. Nuestro criterio es que el valor de los resultados radica en que el modelo se constituye por variables que representan diferentes condicionales del paciente y sus características: demográficas (edad), comorbilidad (HTA), biomarcadores de la inflamación (INL, LDH) y estado de oxigenación $\left(\mathrm{SaO}_{2}\right)$, lo que de alguna manera pudiera hacer más integral la evaluación del médico tratante.

Otros modelos han sido construidos para predecir el riesgo de severidad en la COVID-19. Ji D. et al., (2020) propuso la puntuación CALL (acrónimo en inglés) que incluye cuatro variables en el score: comorbilidad, edad, LDH y recuento de linfocitos; con las cuales también construyeron un nomograma. En este modelo las variables cualitativas fueron dicotomizadas para los resultados, y no se incluyen variables relacionadas con la oxigenación. Nuestro nomograma representa las variables cualitativas de manera continua, permitiendo tener en el gráfico una localización exacta de los valores determinados para cada una de ellas.

El estudio realizado en 2 hospitales chinos por Gong et al., (2020) elaboró un nomograma que incluye un indicador clínico y seis de laboratorio: edad, LDH, proteína 
C reactiva, coeficiente de variación del ancho de distribución de glóbulos rojos, nitrógeno ureico, albúmina, y bilirrubina directa. Según los autores, este modelo es capaz de identificar precozmente a pacientes severos con COVID-19. Este modelo incluye la edad como variable demográfica, sin embrago a diferencia del anterior no tiene en cuenta la comorbilidad, y predominan las variables de laboratorio. Cabe pensar que en este caso se necesita un laboratorio clínico que analice todos los exámenes comprendidos en el nomograma para su implementación. Nuestro nomograma solo incluye como variables de laboratorio el INL que se obtiene fácilmente a partir del hemograma, y la LDH.

Zhou et al., (2020) desarrolló un modelo predictivo basado en la construcción de un nomograma en el cual se incluyeron siete predictores: temperatura corporal al ingreso, tos, disnea, HTA, enfermedad cardiovascular, insuficiencia hepática crónica y enfermedad renal crónica. A diferencia del nuestro, no se incluyeron variables de laboratorio, gasométricas, ni radiológicas.

Liang et al., (2020) también realizó un estudio con el cual desarrolló una escala clínica para predecir enfermedad crítica en pacientes con COVID-19. Diez variables resultaron predictores independientes y fueron incluidas en la escala de riesgo: anomalías en la radiografía de tórax, edad, hemoptisis, disnea, alteración de la consciencia, número de comorbilidades, historia de cáncer, INL, LDH, y bilirrubina directa. Este modelo se caracteriza por la gran cantidad de variables que tiene en cuenta: la radiografía del tórax, una variable demográfica, cinco variables clínicas, tres de laboratorio.

Ninguno de los modelos analizados anteriormente tiene en cuenta la oxigenación como predictor de severidad, sin embargo, Gupta et al., (2020) reconstruyó un modelo a partir de 22 modelos pronósticos en pacientes hospitalizados por COVID-19. Encontró que el predictor con mayor poder de discriminación para el deterioro en el hospital, fue la saturación de oxígeno. Concluye que el valor de la saturación de oxígeno basal y la edad son fuertes predictores de deterioro y mortalidad hospitalaria respectivamente, lo cual reafirma nuestros resultados.

Si bien todos los grupos de edad corren el riesgo de contraer la COVID-19, en el caso de las personas de edad avanzada el riesgo de morir o de enfermar gravemente tras la infección es significativamente mayor, nuestro modelo incluye la edad como una de las variables que predice severidad en pacientes con COVID-19.

Cecconi et al., (2020); Imam et al., (2020); Grasselli et al., (2020); Goyal et al., (2020) se han referido a la edad como variable demográfica con mayor asociación a la severidad y como mayor predictor de mortalidad en pacientes con COVID-19, por lo cual ha sido propuesta para formar parte de varias escalas clínicas de severidad.

Los reportes de la Organización de las Naciones Unidas (2020), indican que se ha llegado a estimar que el $66 \%$ de las personas de 70 años o más tiene al menos una comorbilidad, 
lo que incrementa el riesgo de que sufran los efectos graves de la COVID-19. Los informes a inicios de la pandemia estimaban que más del $95 \%$ de los fallecidos por COVID-19 en Europa eran personas de 60 ańos en adelante. En los Estados Unidos, el 80 \% de las muertes correspondían a adultos con una edad superior a los 65 años, y en China, aproximadamente el $80 \%$ de los fallecidos eran adultos mayores de 60 años.

Varios modelos predictivos tienen incluida la comorbilidad o alguna de ellas. Según nuestros resultados la HTA fue la comorbilidad con mayor poder predictivo en la cohorte de pacientes estudiados.

Ciertamente las comorbilidades se han asociado a un incremento en el riesgo de infección y además a un peor pronóstico y mortalidad debido al incremento de la severidad del daño pulmonar. En la serie reportada por Schiffrin et al., (2020) las comorbilidades más comunes fueron hipertensión arterial, diabetes mellitus y enfermedad de las arterias coronarias.

Las estadísticas actuales en la COVID-19 refieren que la hipertensión arterial es una de las comorbilidades más comunes en los pacientes. Kamyshnyi et al., (2020) argumenta que el hecho de padecer esta enfermedad puede empeorar la evolución y aumentar el riesgo de progresión e ingreso en la unidad de cuidados intensivos. Se han estudiado los mecanismos por los cuales la HTA contribuye al mal pronóstico de la COVID-19, y aún no están claros. Esta asociación puede estar relacionada con el papel de la enzima convertidora de angiotensina 2 en la fisiopatología de la hipertensión arterial, involucrada también en la fisiopatología de la infección por COVID-19. Otro mecanismo es la disregulación del sistema inmune que da lugar a la tormenta de citoquinas, mediada por una respuesta desequilibrada de los subtipos de células $\mathrm{T}$ helper.

Guan et al., (2020a) en un estudio que incluyó 1590 pacientes, concluyó que la hipertensión arterial era la comorbilidad más prevalente $(16,9 \%)$, seguida de diabetes $(8,2 \%)$, las enfermedades cardiovasculares $(3,7 \%)$, las enfermedades cerebrovasculares $(1,9 \%)$, la EPOC $(1,5 \%)$ y la malignidad $(1,1 \%)$.

Este mismo autor, Guan et al., (2020b)en otro estudio de 1099 pacientes encontró que la HTA fue la condición de mayor prevalencia para determinar ingreso en la UCI, uso de ventilación mecánica; o muerte, así como la posibilidad de desarrollar enfermedad severa.

Yang et al., (2020) en un meta-análisis incluyó 46248 pacientes infectados por COVID-19, y encontró que la comorbilidad más prevalente fue la hipertensión (media \pm SD 17士7\%; IC del 95\%: 14-22\%). Además, la HTA y la enfermedad cardiovascular fueron las comorbilidades que más se asociaron significativamente a la severidad, OR 2,36 (IC del 95\%: 1,46-3,83) y OR 3,42 (IC del 95\%: 1,88-6,22), respectivamente.

La respuesta inflamatoria, la tormenta de citoquinas y la evasión viral a la respuesta celular inmune juegan un rol central en la progresión y severidad. Diversas anormalidades 
de laboratorio han sido descritas como asociadas a una evolución no favorable en pacientes con COVID-19 (Lippi \& Plebani, 2020).

En nuestro estudio la LDH y el INL fueron las variables de laboratorio con mayor poder predictivo, ambas como expresión de la respuesta inflamatoria sistémica que se produce durante la infección por COVID-19.

Szarpak et al., (2020) en una revisión sistemática y un meta análisis determinó la utilidad de la LDH como predictor de severidad en pacientes con COVID-19. Se revisaron 28 investigaciones que tuvieron en cuenta los niveles de $\mathrm{LDH}$ en pacientes severos y no severos. Observó que valores elevados de $\mathrm{LDH}$ fueron estadísticamente significativos en pacientes que ingresaron en la UCI en relación a los que no ingresaron $(\mathrm{p}<0.001)$. Esta diferencia también fue altamente significativa en pacientes fallecidos en relación a los no fallecidos, Se concluyó que los niveles de $\mathrm{LDH}$ pueden ser útiles como biomarcador predictor de severidad y de supervivencia en pacientes con COVID-19.

Henry et al.,(2020) analizó 1532 pacientes con el objetivo de evaluar la asociación entre los niveles elevados de LDH determinados precozmente y la evolución posterior durante la hospitalización. Los valores elevados de $\mathrm{LDH}$ estuvieron asociados con un incremento de 6 y 16 veces más con la probabilidad de severidad y mortalidad respectivamente.

Desde hace varios años Zahorec, (2001) identificó la relación neutrófilos/linfocitos como un marcador de inflamación en pacientes críticos. Desde entonces se ha venido estudiando su utilidad en diversas enfermedades incluyendo la sepsis, siendo su aplicación en otras enfermedades cada vez más extendida.

Según Kermali et al., (2020)en la COVID-19 el índiceneutrófilos/linfocitos (INL) junto a otros biomarcadores, es un indicador considerado como predictor de severidad. Song et al., (2020); Zhouet al., (2020)concuerdan en que este índice elevado al ingreso, es un factor de riesgo a corto plazo de progresión a neumonía severa, confirmándose su valor pronóstico en la COVID-19.

Tatum et al., (2020) concluyó que este índice se relaciona con el riesgo de ingreso en la UCI, uso de soporte ventilatorio y mortalidad hospitalaria, determinando un punto de corte óptimo de 4.94 según el área bajo la curva $R O C$.

\section{CONCLUSIONES}

Nuestro nomograma constituye un instrumento de gran utilidad para identificar precozmente los pacientes con riesgo de progresar a formas severas de COVID-19. De esta manera, facilita una mejor estratificación y adecuación de tratamientos oportunos, capaces de frenar la progresión de la enfermedad. 


\section{CONSIDERACIONES ÉTICAS}

Esta investigación fue aprobada por el Comité de Ética de nuestra institución y respeta todas las normas establecidas para las publicaciones médicas.

\section{CONFLICTO DE INTERÉS}

Los autores declaran que no existen conflictos de intereses en esta publicación.

\section{REFERENCIAS}

Beigel, J. H., Tomashek, K. M., \& Dodd, L. E. (2020). Remdesivir for the Treatment of Covid-19 - Preliminary Report. Reply. The New England journal of medicine, 383(10): 994. Disponible en: https://doi.org/10.1056/NEJMc2022236

Cecconi, M., Piovani, D., Brunetta, E., Aghemo, A., Greco, M., Ciccarelli, M., Angelini, C., Voza, A., Omodei, P., Vespa, E., Pugliese, N., Parigi, T. L., Folci, M., Danese, S., \& Bonovas, S. (2020). Early Predictors of Clinical Deterioration in a Cohort of 239 Patients Hospitalized for Covid-19 Infection in Lombardy, Italy. Journal of clinical medicine, 9(5): 1548. Disponible en: https://doi.org/10.3390/jcm9051548

Chen, N., Zhou, M., Dong, X., Qu, J., Gong, F., Han, Y., Qiu, Y., Wang, J., Liu, Y., Wei, Y., Xia, J., Yu, T., Zhang, X., \& Zhang, L. (2020). Epidemiological and clinical characteristics of 99 cases of 2019 novel coronavirus pneumonia in Wuhan, China: a descriptive study. Lancet (London, England), 395(10223): 507-513. Disponible en: https://doi.org/10.1016/S0140-6736(20)30211-7

Gong, J., Ou, J., Qiu, X., Jie, Y., Chen, Y., Yuan, L., Cao, J., Tan, M., Xu, W., Zheng, F., Shi, Y., \& Hu, B. (2020). A Tool for Early Prediction of Severe Coronavirus Disease 2019 (COVID-19): A Multicenter Study Using the Risk Nomogram in Wuhan and Guangdong, China. Clinical infectious diseases : an official publication of the Infectious Diseases Society of America, 71(15): 833-840. Disponible en: https:// doi.org/10.1093/cid/ciaa443

Goyal, P., Choi, J. J., Pinheiro, L. C., Schenck, E. J., Chen, R., Jabri, A., Satlin, M. J., Campion, T. R., Jr, Nahid, M., Ringel, J. B., Hoffman, K. L., Alshak, M. N., Li, H. A., Wehmeyer, G. T., Rajan, M., Reshetnyak, E., Hupert, N., Horn, E. M., Martinez, F. J., Gulick, R. M., ... Safford, M. M. (2020). Clinical Characteristics of Covid-19 in New York City. The New England journal of medicine, 382(24): 2372-2374. Disponible en: https://doi.org/10.1056/NEJMc2010419 
Grasselli, G., Zangrillo, A., Zanella, A., Antonelli, M., Cabrini, L., Castelli, A., Cereda, D., Coluccello, A., Foti, G., Fumagalli, R., Iotti, G., Latronico, N., Lorini, L., Merler, S., Natalini, G., Piatti, A., Ranieri, M. V., Scandroglio, A. M., Storti, E., Cecconi, M., ... COVID-19 Lombardy ICU Network (2020). Baseline Characteristics and Outcomes of 1591 Patients Infected With SARS-CoV-2 Admitted to ICUs of the Lombardy Region, Italy. JAMA, 323(16): 1574-1581. Disponible en: https://doi. org/10.1001/jama.2020.5394

Guan, W. J., Liang, W. H., Zhao, Y., Liang, H. R., Chen, Z. S., Li, Y. M., Liu, X. Q., Chen, R. C., Tang, C. L., Wang, T., Ou, C. Q., Li, L., Chen, P. Y., Sang, L., Wang, W., Li, J. F., Li, C. C., Ou, L. M., Cheng, B., Xiong, S., ... China Medical Treatment Expert Group for COVID-19 (2020a). Comorbidity and its impact on 1590 patients with COVID-19 in China: a nationwide analysis. The European respiratory journal, 55(5): 2000547. Disponible en: https://doi.org/10.1183/13993003.00547-2020

Guan, W. J., Ni, Z. Y., Hu, Y., Liang, W. H., Ou, C. Q., He, J. X., Liu, L., Shan, H., Lei, C. L., Hui, D., Du, B., Li, L. J., Zeng, G., Yuen, K. Y., Chen, R. C., Tang, C. L., Wang, T., Chen, P. Y., Xiang, J., Li, S. Y., ... China Medical Treatment Expert Group for Covid-19 (2020b). Clinical Characteristics of Coronavirus Disease 2019 in China. The New England journal of medicine, 382(18): 1708-1720. Disponible en: https://doi.org/10.1056/NEJMoa2002032

Gupta, R. K., Marks, M., Samuels, T., Luintel, A., Rampling, T., Chowdhury, H., Quartagno, M., Nair, A., Lipman, M., Abubakar, I., van Smeden, M., Wong, W. K., Williams, B., \& Noursadeghi, M., (2020). Systematic evaluation and external validation of 22 prognostic models among hospitalised adults with COVID-19: an observational cohort study. The European respiratory journal, 56(6): 2003498. Disponible en: https://doi.org/10.1183/13993003.03498-2020

Henry, B. M., Aggarwal, G., Wong, J., Benoit, S., Vikse, J., Plebani, M., \& Lippi, G. (2020). Lactate dehydrogenase levels predict coronavirus disease 2019 (COVID-19) severity and mortality: A pooled analysis. The American journal of emergency medicine, 38(9): 1722-1726. Disponible en: https://doi.org/10.1016/j.ajem.2020.05.073.

Horby, P., Lim, W. S., Emberson, J. R., Mafham, M., Bell, J. L., Linsell, L., Staplin, N., Brightling, C., Ustianowski, A., Elmahi, E., Prudon, B., Green, C., Felton, T., Chadwick, D., Rege, K., Fegan, C., Chappell, L. C., Faust, S. N., Jaki, T., ... Landray, M. J. (2021). Dexamethasone in Hospitalized Patients with Covid-19. The New England journal of medicine, 384(8): 693-704. Disponible en:https://doi. org/10.1056/NEJMoa2021436

Imam, Z., Odish, F., Gill, I., O’Connor, D., Armstrong, J., Vanood, A., Ibironke, O., Hanna, A., Ranski, A., \& Halalau, A. (2020). Older age and comorbidity are 
independent mortality predictors in a large cohort of 1305 COVID-19 patients in Michigan, United States. Journal of internal medicine, 288(4): 469-476. Disponible en: https://doi.org/10.1111/joim.13119

Ji, D., Zhang, D., Xu, J., Chen, Z., Yang, T., Zhao, P., Chen, G., Cheng, G., Wang, Y., Bi, J., Tan, L., Lau, G., \& Qin, E. (2020). Prediction for Progression Risk in Patients With COVID-19 Pneumonia: The CALL Score. Clinical infectious diseases : an official publication of the Infectious Diseases Society of America, 71(6); 1393-1399. Disponible en: https://doi.org/10.1093/cid/ciaa414

Ji, W., Wang, W., Zhao, X. and Zai, J.,\&Wong K. (2020). Cross-species transmission of the newly identified coronavirus 2019-nCoV. Journal of medical virology, 92: 433440. https://doi.org/10.1002/jmv.25682

Kamyshnyi, A., Krynytska, I., Matskevych, V., Marushchak, M., \& Lushchak, O. (2020). Arterial Hypertension as a Risk Comorbidity Associated with COVID-19 Pathology. International journal of hypertension, 2020, 8019360. Disponible en: https://doi. org/10.1155/2020/8019360

Kermali, M., Khalsa, R. K., Pillai, K., Ismail, Z., \& Harky, A. (2020). The role of biomarkers in diagnosis of COVID-19 - A systematic review. Life sciences, 254, 117788. Disponible en: https://doi.org/10.1016/j.lfs.2020.117788.

Liang, W., Liang, H., Ou, L., Chen, B., Chen, A., Li, C., Li, Y., Guan, W., Sang, L., Lu, J., Xu, Y., Chen, G., Guo, H., Guo, J., Chen, Z., Zhao, Y., Li, S., Zhang, N., Zhong, N., He, J., ... China Medical Treatment Expert Group for COVID-19 (2020). Development and Validation of a Clinical Risk Score to Predict the Occurrence of Critical Illness in Hospitalized Patients With COVID-19. JAMA internal medicine, 180(8): 1081-1089. Disponible en: https://doi.org/10.1001/ jamainternmed.2020.2033

Lippi, G., \& Plebani, M. (2020). Laboratory abnormalities in patients with COVID-2019 infection. Clinical chemistry and laboratory medicine, 58(7): 1131-1134. Disponible en: https://doi.org/10.1515/cclm-2020-0198

Lipsitch, M., Swerdlow, D. L., \& Finelli, L. (2020). Defining the Epidemiology of Covid-19 - Studies Needed. The New England journal of medicine, 382(13): 1194-1196. Disponible en: https://doi.org/10.1056/NEJMp2002125

Lu, H., Stratton, C. \& Tang, Y., 2020. Outbreak of pneumonia of unknown etiology in Wuhan, China: The mystery and the miracle. Journal of medical virology, 92(4): 401-402. Disponible en: https://doi.org/10.1002/jmv.25678.

Naciones Unidas. (2020) Informe de políticas: Los efectos de laCOVID-19 en laspersonas de edad. New York: Naciones Unidas; Disponible en: https://www.un.org/sites/un2. un.org/files/old_persons_spanish.pdf 
Schiffrin, E. L., Flack, J. M., Ito, S., Muntner, P., \& Webb, R. C. (2020). Hypertension and COVID-19. American journal of hypertension, 33(5): 373-374. Disponible en: https://doi.org/10.1093/ajh/hpaa057

Singer, M., Deutschman, C. S., Seymour, C. W., Shankar-Hari, M., Annane, D., Bauer, M., Bellomo, R., Bernard, G. R., Chiche, J. D., Coopersmith, C. M., Hotchkiss, R. S., Levy, M. M., Marshall, J. C., Martin, G. S., Opal, S. M., Rubenfeld, G. D., van der Poll, T., Vincent, J. L., \& Angus, D. C. (2016). The Third International Consensus Definitions for Sepsis and Septic Shock (Sepsis-3). JAMA, 315(8): 801810. Disponible en: https://doi.org/10.1001/jama.2016.0287

Song, C., Xu, J., He, J., \& Lu, Y. (2020). COVID-19 early warning score: a multi-parameter screening tool to identify highly suspected patients. medRxiv.Disponible en: https:// doi.org/10.1101/2020.03.05.20031906.

Song, F., (2020). Emerging 2019 Novel Coronavirus (2019-nCoV) Pneumonia. Radiology, 295(1): 210-217. Disponible en: http://doi: 10.1148/radiol.2020209021

Szarpak, L., Ruetzler, K., Safiejko, K., Hampel, M., Pruc, M., Kanczuga-Koda, L., Filipiak, K. J., \& Jaguszewski, M. J. (2020). Lactate dehydrogenase level as a COVID-19 severity marker. The American journal of emergency medicine, S0735-6757(20)31034-2. Advance online publication. Disponible en: https://doi. org/10.1016/j.ajem.2020.11.025.

Tatum, D., Taghavi, S., Houghton, A., Stover, J., Toraih, E., \& Duchesne, J. (2020). Neutrophil-to-Lymphocyte Ratio and Outcomes in Louisiana COVID-19 Patients. Shock (Augusta, Ga.), 54(5): 652-658. Disponible en: https://doi.org/10.1097/ SHK.0000000000001585.

Vélez, M., Velásquez Salazar, P., Acosta-Reyes, J., Vera-Giraldo, C., Franco, J. and Jiménez, C., 2020. Factores clínicos pronósticos de enfermedad grave y mortalidad en pacientes con COVID-19. [ebook] Antioquia: Unidad de Evidencia y Deliberación para la Toma de Decisiones (UNED), p.6. Disponible en: https://es.cochrane.org/ sites/es.cochrane.org/files/public/uploads/COVID-19/udea-uned_sintesisrapida_ covid-19_pronostico_22abril2020.pdf

Wynants, L., Van Calster, B., Collins, G. S., Riley, R. D., Heinze, G., Schuit, E., Bonten, M., Dahly, D. L., Damen, J., Debray, T., de Jong, V., De Vos, M., Dhiman, P., Haller, M. C., Harhay, M. O., Henckaerts, L., Heus, P., Kammer, M., Kreuzberger, N., Lohmann, A., ...\&Smeden, M. (2020). Prediction models for diagnosis and prognosis of covid-19 infection: systematic review and critical appraisal. BMJ (Clinical research ed.), 369, m1328. Disponible en: https://doi.org/10.1136/bmj. m1328 
Yang, J., Zheng, Y., Gou, X., Pu, K., Chen, Z., Guo, Q., Ji, R., Wang, H., Wang, Y., \& Zhou, Y. (2020). Prevalence of comorbidities and its effects in patients infected with SARSCoV-2: a systematic review and meta-analysis. International journal of infectious diseases : IJID : official publication of the International Society for Infectious Diseases, 94: 91-95. Disponible en: https://doi.org/10.1016/j.ijid.2020.03.017

Yang, X., Yu, Y., Xu, J., Shu, H., Xia, J., Liu, H., Wu, Y., Zhang, L., Yu, Z., Fang, M., Yu, T., Wang, Y., Pan, S., Zou, X., Yuan, S., \& Shang, Y. (2020). Clinical course and outcomes of critically ill patients with SARS-CoV-2 pneumonia in Wuhan, China: a single-centered, retrospective, observational study. The Lancet. Respiratory medicine, 8(5): 475-481. Disponible en: https://doi.org/10.1016/S2213-2600(20)30079-5

Zahorec R. (2001). Ratio of neutrophil to lymphocyte counts--rapid and simple parameter of systemic inflammation and stress in critically ill. Bratislavske lekarske listy, 102(1): 5-14.

Zhou, F., Yu, T., Du, R., Fan, G., Liu, Y., Liu, Z., Xiang, J., Wang, Y., Song, B., Gu, X., Guan, L., Wei, Y., Li, H., Wu, X., Xu, J., Tu, S., Zhang, Y., Chen, H., \& Cao, B. (2020). Clinical course and risk factors for mortality of adult inpatients with COVID-19 in Wuhan, China: a retrospective cohort study. Lancet (London, England), 395(10229): 1054-1062. Disponible en: https://doi.org/10.1016/ S0140-6736(20)30566-3

Zhou, Y., He, Y., Yang, H., Yu, H., Wang, T., Chen, Z., Yao, R., \& Liang, Z. (2020). Development and validation a nomogram for predicting the risk of severe COVID-19: A multi-center study in Sichuan, China. PloS one, 15(5): e0233328. Disponible en: https://doi.org/10.1371/journal.pone.0233328

Zhou, Y., Yang, Z., Guo, Y., Geng, S., Gao, S., Ye, S., Hu, Y., and Wang, Y. (2020). A New Predictor of Disease Severity in Patients with COVID-19 in Wuhan, China. medRxiv. Disponible en: https://doi.org/10.1101/2020.03.24.20042119.

Recibido: 31 de mayo de 2021 Aceptado: 05 de julio de 2021 